\title{
Low TLR7 gene expression in atherosclerotic plaques is associated with major adverse cardio- and cerebrovascular events
}

Karadimou, Glykeria; Folkersen, Lasse; Berg, Martin; Perisic, Ljubica; Discacciati, Andrea; Roy, Joy; Hansson, Göran K; Persson, Jonas; Paulsson-Berne, Gabrielle

Published in:

Cardiovascular Research

Link to article, DOI:

$10.1093 / \mathrm{cvr} / \mathrm{cvw} 231$

Publication date:

2016

Document Version

Peer reviewed version

Link back to DTU Orbit

Citation (APA):

Karadimou, G., Folkersen, L., Berg, M., Perisic, L., Discacciati, A., Roy, J., Hansson, G. K., Persson, J., \& Paulsson-Berne, G. (2016). Low TLR7 gene expression in atherosclerotic plaques is associated with major adverse cardio- and cerebrovascular events. Cardiovascular Research, 113(1), 30-39.

https://doi.org/10.1093/cvr/cvw231

\section{General rights}

Copyright and moral rights for the publications made accessible in the public portal are retained by the authors and/or other copyright owners and it is a condition of accessing publications that users recognise and abide by the legal requirements associated with these rights.

- Users may download and print one copy of any publication from the public portal for the purpose of private study or research.

- You may not further distribute the material or use it for any profit-making activity or commercial gain

- You may freely distribute the URL identifying the publication in the public portal 


\section{Low TLR7 gene expression in atherosclerotic plaques is associated}

\section{with major adverse cardio- and cerebrovascular events}

Running title: "Low TLR7 gene expression is associated with MACCE"

Glykeria Karadimou*1, Lasse Folkersen ${ }^{* 1,2}$, Martin Berg ${ }^{1}$, Ljubica Perisic ${ }^{3}$, Andrea Discacciati $^{4}$ Joy Roy $^{3}$, Göran K Hansson ${ }^{1}$, Jonas Persson ${ }^{1,5}$, and Gabrielle Paulsson-Berne ${ }^{1}$

${ }^{1}$ Unit of Cardiovascular Medicine, Department of Medicine, Karolinska Institutet \& Karolinska University Hospital, Stockholm, Sweden

${ }^{2}$ Center for Biological Sequence Analysis, Department of Systems Biology, Technical University of Denmark, Denmark

${ }^{3}$ Department of Molecular Medicine and Surgery, Karolinska University Hospital, Karolinska Institutet, Stockholm, Sweden

${ }^{4}$ Unit of Biostatistics, Institute of Environmental Medicine, Karolinska Institutet, Stockholm, Sweden

${ }^{5}$ Division of Cardiovascular Medicine, Department of Clinical Sciences, Karolinska Institutet, Danderyd University Hospital, Stockholm, Sweden

Corresponding author:

Gabrielle Paulsson-Berne, telephone +46 8 51776223, fax number +46 8313147

e-mail Gabrielle.Berne@ki.se

*Contributed equal to this work

Word count 6569

(C) The Author 2016. Published by Oxford University Press on behalf of the European Society of Cardiology. This is an Open Access article distributed under the terms of the Creative Commons Attribution NonCommercial License (http://creativecommons.org/licenses/by-nc/4.0/), which permits non-commercial reuse, distribution, and reproduction in any medium, provided the original work is properly cited. For commercial re-use, please contact journals.permissions@oup.com 


\section{ABSTRACT}

\section{Aims}

Processes in the development of atherosclerotic lesions can lead to plaque rupture or erosion, which can in turn elicit myocardial infarction or ischaemic stroke. The aims of this study were to determine whether Toll-like receptor 7 (TLR7) gene expression levels influence patient outcome and to explore the mechanisms linked to TLR7 expression in atherosclerosis.

\section{Methods and results}

Atherosclerotic plaques were removed by carotid endarterectomy (CEA) and subjected to gene array expression analysis $(n=123)$. Increased levels of TLR7 transcript in the plaques were associated with better outcome in a follow-up study over a maximum of 8 years. Patients with higher TLR7 transcript levels had a lower risk of experiencing major cardiovascular and cerebrovascular events (MACCE) during the follow-up period after CEA (hazard ratio: 2.38, $\mathrm{p}=0.012,95 \%$ CI 1.21-4.67). TLR7 was expressed in all plaques by T cells, macrophages and endothelial cells in capillaries, as shown by immunohistochemistry. In short-term tissue cultures, ex vivo treatment of plaques with the TLR7 ligand imiquimod elicited dose-dependent secretion of IL-10, TNF- $\alpha$, GM-CSF, and IL-12/IL-23p40. This secretion was blocked with a TLR7 inhibitor. Immunofluorescent tissue analysis after TLR7 stimulation showed IL-10 expression in T cells, macrophages and vascular smooth muscle cells. TLR7 mRNA levels in the plaques were correlated with IL-10 receptor $(\mathrm{r}=0.4031, \mathrm{p}<0.0001)$ and GM-CSF receptor A $(\mathrm{r}=0.4354, \mathrm{p}<0.0001)$ transcripts.

\section{Conclusion}

These findings demonstrate that TLR7 is abundantly expressed in human atherosclerotic plaques. TLR7 ligation elicits the secretion of pro-inflammatory and anti-inflammatory cytokines, and high TLR7 expression in plaques is associated with better patient outcome, 
suggesting that TLR7 is a potential therapeutic target for prevention of complications of atherosclerosis.

Keywords: atherosclerosis, inflammation, carotid stenosis, cytokine, IL-10 


\section{INTRODUCTION}

Atherosclerosis is the major cause of myocardial infarction and stroke. Atherosclerotic lesions have complex structures and show extensive infiltration by immune cells, such as macrophages and T cells. ${ }^{1}$ Inflammatory components have been implicated in plaque rupture ${ }^{2}$, which can lead to myocardial infarction or stroke.

Many inflammatory mediators, such as innate and adaptive cytokines, chemokines, and receptors, have significant functions in atherosclerosis. ${ }^{3}$ Toll-Like Receptors (TLRs) were originally found to recognize conserved motifs in pathogens, called pathogen-associated molecular patterns (PAMPs), such as the lipopolysaccharides that are secreted by bacteria. Recently, TLRs have also been reported to recognize endogenous host molecules that are released during cell death and injury, referred to as damage-associated molecular patterns (DAMPs). ${ }^{4}$

Currently, several TLRs in humans have been identified; some localize to the cell surface, whereas others are found intracellularly and span endosomal membranes. ${ }^{4}{ }^{5}$ Initial studies on TLR function in atherosclerosis have demonstrated that TLR4 activation in mouse models increases the progression of the disease and a genetic variant in humans heightens the CVD risk. ${ }^{5}$ Recent studies have shown that TLR2 activation leads to endothelial erosion and increases the risk of thrombotic complications. ${ }^{6}$ However, Salagianni et al have recently reported that TLR7 protects against atherosclerosis in experimental models of the disease. ${ }^{7}$

A better molecular understanding of the late stages of atherosclerosis is paramount for improving several aspects of disease management, including its diagnosis and prevention. The

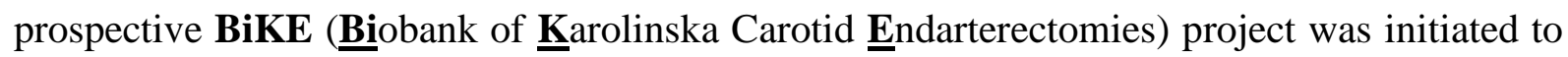
examine the molecular mechanisms underlying the formation of atherosclerotic plaques. ${ }^{8,9}$ Patients scheduled for carotid endarterectomy (CEA) on Karolinska Hospital were 
consecutively enrolled and transcript profiles of the removed plaques were analysed. Transcript profile analysis is a sensitive method of detecting and mapping ongoing processes in plaques. In a previous study, we have examined whether transcript signatures from the removed plaques correlated with subsequent events in the patients. Our study demonstrated, as a proof of concept, that the inclusion of gene expression profiles of the carotid plaques that are collected during endarterectomy improves the prediction of adverse events, compared with the use of only the classical risk markers in patients with atherosclerosis. ${ }^{10}$

We set up an analysis with the aim of linking specific transcripts in atherosclerotic lesions to patient outcome. One candidate of interest has been identified: TLR7. ${ }^{7}$ In this study we found and validated that high TLR7 levels correlate with better patient outcome in cases of severe atherosclerosis. We also conducted a thorough protein-level investigation of the processes through which this gene affects the future risk of cardiovascular events in CEA patients. 


\section{MATERIALS AND METHODS}

\section{Sample collection}

Materials from the Biobank of Karolinska carotid Endarterectomies (BiKE) project were obtained after all participants had provided informed written consent, per the Declaration of Helsinki and the Karolinska Institute ethics committee. The study included a total of 179 atherosclerotic plaque tissue samples from patients who underwent CEA at Karolinska University Hospital, Stockholm, Sweden. The patient characteristics are summarized in Table 1. The majority of the patients underwent standard endarterectomy under sedation and local anaesthesia. After being rinsed, the plaque was frozen instantly on dry ice for subsequent isolation of RNA. For immunohistochemical or immunofluorescent analysis plaque was paraffin-imbedded for morphological analysis.

\section{RNA isolation}

Total RNA was isolated using an RNeasy Mini kit followed by DNase treatment according to the manufacturer's instructions. RNA quality was analysed on an Agilent 2100 Bioanalyzer, and RNA concentration was measured with a NanoDrop spectrophotometer. Lowconcentration and low-quality samples were omitted.

\section{Follow-up and definitions of events}

The BiKE database ${ }^{8-10}$ was merged with the Swedish Hospital Discharge Register and the Swedish Cause of Death Register for the follow-up of adverse cardiovascular, cerebrovascular, and vascular events. Major adverse cardio- and cerebrovascular events (MACCE) were defined as the combined incidence of myocardial infarction, stroke, percutaneous coronary intervention, coronary artery bypass grafting, endovascular intervention of peripheral arteries and open surgery on peripheral arteries, as reported to the registers listed above. Ischaemic events were 
defined as fatal or nonfatal myocardial infarction or ischaemic stroke. The retrieval of myocardial infarction and stroke incidence data from the Swedish Hospital Discharge Register and the Swedish Cause of Death Register is a reliable, validated alternative to the use of revised hospital discharge and death certificates. ${ }^{11,12}$ Total number of MACCE and MI events in the microarray discovery cohort was 34 and 24 respectively. In the qPCR validation cohort 47 MACCE and 33 MI events occurred.

\section{Gene expression microarrays}

RNA samples were hybridized and scanned at the Karolinska Institute Affymetrix core facility using Affymetrix HG-U133 plus 2.0 arrays. The resulting cel files were preprocessed by RMA normalization $^{13}$, as implemented in the Affymetrix Power Tools 1.10.2 apt-probeset-summarize package. Further information is described in a previous study. ${ }^{10}$ The number of samples for which both microarray measurements and MACCE information was available was 123 .

\section{Real-time PCR}

Total RNA was reverse-transcribed with SuperScript II reverse transcriptase and random hexamers, using a master mix for all samples in 1 batch. The same amount of total RNA was used for each sample, based on measurements from a NanoDrop 1000 spectrophotometer. TaqMan probes and primers for TLR7 were purchased as assay-on-demand products. PCR was conducted in 4 96-well plates on a 7700 HT fast real-time PCR system. All samples were measured in duplicate. All real-time PCR analyses were performed using the $2^{-\Delta \mathrm{Ct}}$ method, with the mean of all samples used as a calibrator.

\section{Immunohistochemistry}

Serial paraffin sections of human CEA specimens were incubated with primary antibodies to CD3 (pre-diluted), CD8 (1:50), CD163 (pre-diluted), CD68 (1:50) and TLR7 (1:600) overnight 
at $4^{\circ} \mathrm{C}$. Signals were detected with biotinylated secondary antibodies and developed with Vulcan Fast Red.

\section{Immunofluorescence}

Sections were incubated for $1 \mathrm{~h}$ in RT with primary antibodies against CD3 (1:500), CD8 (1:50), CD163 (pre-diluted), CD68 (1:50), FoxP3 (1:200), $\alpha$-actin (1:400). CD31 (1:50) and von Willebrand factor $(1: 2000)$ and overnight at $4^{\circ} \mathrm{C}$ with antibodies to TLR7 (1:600), IL-10 (1:3000) and CD69 (1:50). Sections were blocked with 5\% serum and incubated with the following secondary antibodies: Dylight 488 anti-mouse. Dylight 594 anti-rabbit (1:300), and Alexa 647 anti-goat (1:200). Autofluorescence was blocked with 0.03\% Sudan Black, and DAPI was used for nuclear staining. Sections were analysed with a Leica TCS-SP5 confocal microscope. No differences in staining patterns were observed between consecutive single-, double-, or triple-stained sections.

For IL-10 staining, carotid plaque tissue $(n=1)$ was separated in 4 large pieces. Half of the pieces were stimulated ex-vivo with $12.5 \mu \mathrm{g} / \mathrm{ml}$ IMQ, the unstimulated pieces where used for control. Pieces were collected after $1.5 \mathrm{~h}$ and $8 \mathrm{~h}$. Pieces were fixed in $4 \%$ zinc-formaldehyde solution and embedded in paraffin after dehydration.

\section{Ex vivo human carotid plaque stimulation with TLR7 agonist}

For cytokine release assay: Twelve fresh carotid plaques were obtained from patients undergoing CEA. At the operation day the plaque was cut into small pieces $\left(\sim 2 \mathrm{~mm}^{3}\right)$, and distributed into the wells of a 48-well plate and thereafter pre-incubated for $1 \mathrm{~h}$ in RPMI 1640 medium supplemented with $10 \%$ human serum at $37^{\circ} \mathrm{C}$ in $5 \% \mathrm{CO}_{2}$ before stimulattion with TLR7 agonist imiquimod (IMQ). The following concentrations were used in a dose response challenge $2.5,5$, or $12.5 \mu \mathrm{g} / \mathrm{ml}$ IMQ. Each dose was run in duplicates and the reported values are the average for each concentration. See Supplemental Materials: Reagents for information 
about IMQ. As control $100 \mathrm{ng} / \mathrm{ml}$ lipopolysaccharide (LPS) was used to assure live cells present in the tissue after the mincing. After $20 \mathrm{~h}$ of stimulation, supernatants from the plaques were collected and stored at $-80^{\circ} \mathrm{C}$ for cytokine analysis. Supernatant from unstimulated tissue was used as control. See figure 5 .

\section{Analysis of cytokine secretion}

Cytokine levels in supernatants were measured after ex vivo stimulation using MesoScale technology (MSD). IFN- $\nu$, IL-1 $\beta$, IL-2, IL-4, IL-6, IL-10, IL-12p70, IL-13, and TNF- $\alpha$ were measured with the "Proinflammatory 10-plex panel 1", and GM-CSF, IL-1 $\alpha$, IL-5, IL-7, IL12/IL-23p40, IL-15, IL-16, IL-17A, TNF- $\beta$, and VEGF were measured with a "Cytokine 10plex panel 1" (MSD) according to the manufacturer's protocol. For IL-8, a separate ELISA kit was used. For the cytokine measurements after ex vivo IMQ stimulation of isolated CD4, CD8 and CD14 cells, single cytokine ELISA kits were used for IL-10, TNF- $\alpha$ and GM-CSF.

In vitro blocking assay of TLR7

In separate inhibition experiments eight carotid plaques were used. The minced plaque tissue was pre-incubated for $1 \mathrm{~h}$ with $5 \mu \mathrm{M}$ of a TLR7 inhibitor (ODN 20958) before addition of TLR7 agonist IMQ (12.5 $\mu \mathrm{g} / \mathrm{ml})$. For the cytokine measurements after ex vivo TLR7 inhibition and ELISA kits were used for IL-10, TNF- $\alpha$, GM-CSF and IL-8. See figure 6.

\section{Reagents}

All reagents are listed in the supplemental file.

\section{Statistical analysis}

Statistical analysis was performed using R 3.1.2 (R Foundation, Tables 1 and 2 and Figure 1). Survival was analysed using a Cox regression model and implemented in the survival bioconductor package (version 2.37.7), as described previously. ${ }^{10,14}$ Baseline data in table 1 are 
presented as median [first quartile, third quartile]. In the survival analysis (figure 1), subjects accrued follow-up time from time of CEA until the time of event (MACCE or ischaemic event, depending on the analysis) or censoring, whichever came first. MACCEs and MIs were classified with the ICD-10 codes as described previously. ${ }^{15}$ TLR7 expression values were categorized into tertiles. Cox proportional hazards models were used to estimate hazard ratios (HRs) and 95\% confidence intervals (CIs) for the lowest tertile of TLR7 expression versus the middle and highest tertiles combined. Cox models were further adjusted for age, sex, and LDLcholesterol (Table 2). The proportional hazard assumption was tested by means of Schoenfeld's residuals. No evidence of departure from this assumption was observed. Kaplan-Meier plots were generated using the plot function in the survival package (2.37.7).

The size of the qPCR validation cohort was fixed, but we used the power SurvEpi R-package to calculate that there was 0.76 power to detect the same association that was found in the microarray cohort. Primary data for this calculation was input using the measurements from the microarray cohort. The number of samples that had both qPCR measurements and MACCE information was 133. Fifty-six samples from the qPCR measurements were independent of the microarray dataset.

Mean cytokine secretion $( \pm$ SD) by TLR7 stimulation with different levels of imiquimod $(0$, 2.5, $5,12.5 \mu \mathrm{g} / \mathrm{ml}$ ) are presented in Figure 5 and Table S2. Fixed-effects linear regression was used to test for a linear trend between imiquimod stimulation and mean cytokine secretion (Table S2) performed with Stata release 14.1 (StataCorp, College Station, TX). Inhibition experiments were analysed by one-way paired ANOVA (Figure 6). Graphs and inhibition analysis were performed using Prism (GraphPad Software Inc. version 6.0). Bonferroni correction for multiple testing was used when appropriate. P-values $<0.05$ were considered statistically significant. 


\section{RESULTS}

TLR7 transcript levels in carotid plaques correlate with patient outcome

Transcript profiles of the gene expression data from carotid plaques $(n=123)$ were analysed in samples from the BiKE biobank to determine whether TLR7 levels in plaques correlated with future events and patient outcome. Follow-up data from the clinical registries were extracted for patients who had undergone CEA (Table 1). Patients were followed for median 1410 days / 46 months / 3.8 years with a maximum of 100 months ( 8.3 years), depending on when they entered the study.

Transcript profiles and outcome were compared for patients who experienced major adverse cardio- and cerebrovascular events (MACCE) or ischaemic event (Figure 1, Table 2), and our analysis implicated TLR7 as a strong candidate. Comparing outcome for patients, based on tertile levels of the TLR7 transcript in their plaques, patients in the lowest tertile had a higher risk of encountering a MACCE during the follow-up period after carotid CEA (black line in the Kaplan-Meier plot, Figure 1). Baseline clinical data levels did not differ among the patients in the different tertiles of TLR7 transcript levels (Table 1). The Cox regression hazard ratio for MACCEs (2.38, p=0.012) was not significantly affected by the inclusion of age, sex, and LDL level as risk factors (Table 2). Our analysis show that high TLR7 levels correlate to fewer cardio- and cerebrovascular events.

To verify these results, real-time RT-PCR was performed on an increased number of carotid plaques $(n=133)$, which demonstrated similar results (HR 1.91, $\mathrm{p}=0.027$ ); fewer MACCEs developed over time in patients with higher TLR7 transcript levels (Figure 1b).

TLR7+ cells in human atherosclerotic plaques

Next, we measured TLR7 levels by immunohistochemistry. TLR7 was expressed in all plaques (Figure $2 a, b, e$, and f). The staining pattern indicated that TLR7 was expressed primarily on 
cells of haematopoietic origin. Consecutive sections that were stained with T cell (Figure $2 a$, b) and macrophage (Figure 2c, d) markers showed that TLR7 co-localized with the areas that were infiltrated by these cell types.

To identify the specific cell types that expressed TLR7, we performed double and triple staining using a panel of surface markers analysed by confocal immunofluorescence microscopy. For macrophages, we used CD68 for the M1 subtype (Figure 3a, b) and CD163 for the M2 subtype (Figure $3 c, d$ ). Both macrophage types expressed TLR7. However, several TLR7+ cells in the sections were negative for both markers, indicating that other cell types also expressed TLR7.

TLR7 is differentially expressed between artery-lining endothelium and capillary vessels

Various endothelial cell subtypes differentially expressed TLR7. In the endothelium that lined the carotid lumen, we did not detect any TLR7+ endothelial cells. TLR7+ cells were detected below the endothelium (von Willebrand factor-positive) in clearly separate cells (Figure $3 e, f$ ). However, in the capillary vessels of certain patients, TLR7 co-stained with von Willebrand factor (Figure $3 g, h$ ) in a ring-like pattern typical of capillaries. Signals for $\alpha$-actin, a marker of vascular smooth muscle cells, did not overlap with TLR7 (data not shown). Analysis of medium sized normal arteries (iliaca) indicated that TLR7 expression is not present in lumen endothelium and, only occasionally faintly in the capillaries (figure S1).

\section{Robust TLR7 expression in T cells infiltrating atherosclerotic plaques}

We hypothesized that $\mathrm{T}$ cells constitute a significant TLR7+ cell population and identified them by co-staining for CD3 and CD8 (Figure 4a-d). CD3+CD8- cells were assumed to be CD4+ T cells (Figure $4 c, d$ ), although CD4 was not detected by direct staining. CD4 and CD8 cells were observed in the plaques, often in clusters. CD8+ and CD8- T cells stained robustly for TLR7.

CD69 staining was used to investigate the activation state of the infiltrating T cells. Clusters of activated T cells expressed CD69 and TLR7 (Figure 4e-h). Some regulatory CD4 T cells were 
observed in the plaques, based on FoxP3 expression (Figure 4j-l), and were also positive for TLR7.

Plaques produced cytokines ex vivo in response toTLR7 ligand

To determine the effects of TLR7 activation in plaques, we developed an ex vivo stimulation protocol for plaque tissue. Fresh carotid plaques were cut into small pieces before being treated with the TLR7-specific ligand imiquimod (Figure 5). To verify that plaque cells remained capable of responding to stimuli under the incubation conditions, the plaque tissue was also treated with the TLR4 ligand LPS (data not shown). After incubation with these ligands, the medium was collected and analysed by multiplex ELISA for 20 cytokines (see Materials and Methods).

Notably, the plaque tissue responded to TLR7 ligation by producing IL-10 in a dose-dependent manner. There was also a dose-dependent increase in TNF- $\alpha$, GM-CSF, and IL-12/IL-23p70 after administration of imiquimod (Figure 5). Several cytokines were unaffected by TLR7 stimulation, including IL-2, IL-4, IL-5, IL-6, IL-7, IL-13, and IFN $\gamma$, despite the substantial secretion of these cytokines by plaque tissue regardless of whether ligand was added (Table S2). The concentrations of certain cytokines were low or close to the limit of detection, such as IL-1 $\alpha$ and IL-17 (Table S2).

Inhibition of TLR7 reduces the cytokine secretion

To further investigate the specificity of TLR7 activation, an inhibition step was introduced. The ex vivo protocol for the stimulation of fresh carotid plaque (see above) was repeated with the inclusion of an incubation with a TLR7 inhibitor (see Materials and Methods). This resulted in a strongly reduced release of cytokines (Figure 6). The inhibition was pronounced for IL-10, TNF- $\alpha$ and GM-CSF. Inhibitor only did not affect the cytokine release (data not shown). 
Imiquimod has been used in other studies because it is a TLR7 specific ligand. We sought to investigate the activation level for imiquimod in comparison with other TLR7 ligands. The in vitro system of TLR7-transformed HEK cells was used, and two other ligands, CL307 and R848, were also tested. The comparisons showed a more moderate response to imiquimod than to CL307 and R848 (Figure S2).

\section{IL-10 secretion in plaques and in vitro.}

To trace the cellular origin of the IL-10 secretion within the plaque tissue, two approaches were used: an ex vivo protocol with carotid plaques and an in vitro protocol using PBMCs from patients. First, fresh carotid plaque tissue was incubated with imiquimod for 1.5 or 8 hours and, thereafter, fixed and embedded for immunofluorescent analysis. IL-10 was detected in a portion of both CD163+ macrophages and T cells after the short incubation with the TLR7 ligand, indicating a fast response to stimulation (Figure 7). Another pattern was detected for SMCs, in which IL-10-positive SMCs were detected both in the control samples (untreated tissue) and after incubation with the TLR7 ligand, indicating a basal level of IL-10 production independent of ligand (Figure 7).

In the second approach, PBMCs from patients were used to set up primary cell cultures of $\mathrm{T}$ cells and macrophages. The response to TLR7 ligand in purified leucocyte populations was very low, indicating a clear difference with the complex plaque tissue. Stimulation with the TLR7 ligand showed hardly any induction of cytokines in the CD4+ T cells. Regarding CD8 cells there was a tendency in decreased cytokine release after the TLR7 ligand was added to culture. After challenging the macrophages with the TLR7 ligand, a tendency of increased amount of secreted IL-10 was detected (Figure S3). Neither the response from T cells nor macrophages were statistically significant.

TLR7 transcript levels correlate with cytokine receptor $m R N A$ 
To determine whether TLR7 levels correlated with cytokines or their corresponding receptors in plaques, we extracted the data from our transcript profiles for the cytokine transcripts that were secreted in a dose-dependent manner after TLR7 stimulation. TLR7 levels correlated with cytokine receptors, such as IL-10 receptor $(\mathrm{r}=0,416, \mathrm{p}<0.0001)$ and GM-CSFRA $(\mathrm{r}=0.4354$, $\mathrm{p}<0.0001)$ (see Table S3). 


\section{DISCUSSION}

Large-scale analysis of expression in affected tissue has the potential to identify specific molecular pathological processes as well as to demonstrate the activity of gene products and their links to downstream proteins. Such studies can increase understanding of the individual differences in a diseased population.

Our biobank project, BiKE, has allowed us to extract data from a gene array expression screening of carotid plaques. We sought to identify individual genes that influence outcome in atherosclerotic patients. By using a bioinformatics analysis, in which we correlated transcriptome data from carotid plaques to follow-up data and patient outcome, we linked TLR7 to a better prognosis and the occurrence of fewer ischaemic events. The follow-up data were collected over 8 years. However, this study was not powered to completely exclude complex dependence with other known risk factors. A larger study is needed for that.

Initially, it was surprising that a TLR family member was linked to protective mechanisms in patients, given the previous findings that TLR activation is detrimental in atherosclerosis. ${ }^{5}$ Nevertheless, 2 exceptions have been reported: endosomal TLR3 and TLR7 have been shown to be atheroprotective in experimental mouse models. ${ }^{5,7}$ Additionally, recent publications from other groups have reported TLR7-dependent anti-inflammatory control mechanisms. ${ }^{16,17}$

TLR7 was expressed in all analysed plaques. In our screening of markers for cells that are usually found in the plaque and in healthy intima, we noted a co-expression of TLR7 in infiltrating leukocytes but not in smooth muscle cells or the luminal endothelium. Using immunofluorescence analysis, TLR7 was detected in macrophages and T cells. Recent studies have identified subtypes of $\mathrm{T}$ cells and macrophages that do not necessarily drive pathogenesis but may mediate healing, likely by primarily eliciting anti-inflammatory responses. ${ }^{18,19}$ 
Regarding the downstream effects of TLR7 activation, two recent studies have implicated TLR7 in the control or abatement of inflammation and in the adaptive immune response in both macrophages ${ }^{16}$ and $\mathrm{T}$ cells ${ }^{17}$, cell types which are frequently found in atherosclerotic lesions. In an experimental study of airway inflammation, imiquimod promoted the resolution of inflammation by inducing the lipid mediator resolvin $\mathrm{D}$ in murine macrophages ${ }^{16}$, an effect that has also been observed in vitro in human monocytes. Furthermore, TLR7 induces anergy in CD4+ T cells in HIV-positive patients. ${ }^{17}$. A similar mechanism might exist in atherosclerotic tissue. In contrast to the effects in HIV-positive patients, the effects of this response would be favourable to individuals with heart disease. Whether the same immunosuppressive mechanisms are present in human CD8 T cells is unknown.

Endothelial cells showed differential TLR7 expression in the plaque. No co-expression was found in the endothelium that lines the carotid lumen. However, TLR7 co-stained with von Willebrand factor in capillary vessels. Thus, whether there is general TLR7 activation during new vessel formation or whether this pattern is an atherosclerosis-specific process remains to be determined.

To examine how plaque tissue responds to TLR7 activation, we developed an ex vivo model using plaque immediately after CEA. After stimulation with the synthetic ligand imiquimod ex vivo, several cytokines such as IL-10, showed a dose-dependent response, whereas some cytokines were unchanged. IL-10 is an immune-suppressing cytokine that has been suggested to ameliorate atherosclerosis. ${ }^{20,21}$ These findings indicate that TLR7 signalling supports an antiinflammatory response in atherosclerotic plaques through IL-10.

Conversely, imiquimod induced the dose-dependent secretion of pro-inflammatory cytokines, such as TNF- $\alpha$. The function of GM-CSF in plaques is under debate. We noted a TLR7 liganddependent GM-CSF response. A recent study has demonstrated that GM-CSF functions as a 
growth factor and increases macrophage apoptosis in advanced lesions. ${ }^{22}$ These effects are mediated by IL-23, another cytokine that we detected after TLR7 activation. Several prominent pro-inflammatory cytokines were unaffected by the TLR7 ligand in the plaques, including IFN $\gamma$ and IL-6.

Our ex vivo data suggested that TLR7 activation modulates and fine-tunes the balance between the induction and suppression of inflammation. Although the response to endogenous TLR7 ligands in plaques is unknown, we hypothesize that this equilibrium is tilted in favour of an inflammation-resolving pathway. The first TLR7 specific ligands to be identified were viral, single-stranded RNA, but several mammalian endogenous ligands have recently been identified, such as RNA from apoptotic cells, miRNA and extracellular RNA from necrotic cells $^{23}$. In atherogenesis, RNA from apoptotic and necrotic cells and from tissue damage could be a plausible source of ligands.

We were interested in determining whether the immunosuppressive response originates from $\mathrm{T}$ cells or macrophages. Clusters of CD8+ and CD4+ cells exist in the plaques. FoxP3+CD4 cells constitute a Treg population that is atheroprotective, particularly in experimental settings. Recent data have suggested that there are CD8+ T cells with an immunosuppressive capacity ${ }^{24}$ and that such CD8+ Tregs influence atherosclerosis. ${ }^{24}$. To shed light on these mechanisms, PBMCs from patients undergoing CEA were used for in vitro analysis. However, the in vitro system with purified CD4 T cells, CD8 T cells or macrophages did not at all mount the same strong response in IL-10 secretion as that observed in the plaque tissues examined in the ex vivo protocols. It therefore seems possible that the specific interactions of the cells in the plaques prime the response and that the milieu to elicit fast TLR7 response is enhanced in plaques. Our tissue analysis with confocal immunofluorescence analysis detected IL-10 in T cells and macrophages in the plaques after a short incubation with a TLR7 ligand, which perhaps triggered the activation of the surrounding cells. Together, our data suggest that upon TLR7 
activation, T cells and CD163 macrophages in the plaque quickly respond with a boost of IL10 that is propagated through the tissue and elicits a greater release of IL-10 from VSMCs. This can occur without extra stimulation by a synthetic ligand because IL-10-positive smooth muscle cells are present in human lesions, as has also been previously shown by others. ${ }^{20}$

However, there are some limitations to our study. Our cohort of patients is relatively small, especially when it comes to the material that has undergone transcript analysis. The analysis of TLR7 transcript levels and the correlation to patients' outcome was not powered to completely exclude complex dependence with other known risk factors, a larger study is needed for that. We also note that the statistical significance has some variation between our two technical approaches (eg gene array profiling versus real-time PCR) to collect transcript data. Therefore further validation of the data will shed light in these questions.

In conclusion, we have identified TLR7 as one marker of better outcome in patients with severe atherosclerosis. In patients who undergo CEA, increased levels of the TLR7 transcript in the removed plaque is associated with fewer adverse cardiovascular events. TLR7 is expressed in $\mathrm{T}$ cells and macrophages within the plaque and in capillary endothelial cells. When plaque tissue is challenged with the TLR7 ligand imiquimod ex vivo, IL-10 is secreted, one of the major anti-inflammatory cytokines, as well as the proinflammatory mediator TNF- $\alpha$. Collectively, these data implicate that TLR7 may be part in modulating inflammatory responses in atherosclerosis by counteracting effect of proinflammatory cytokines.

\section{FUNDING}

This work was supported by grants from the Swedish Research Council, Swedish Heart-Lung Foundation, and the Foundation for Strategic Research in Sweden. EU-FP7/2007-2013, VIA grant $n^{\circ} 603131$.

\section{DISCLOSURES}


None 


\section{REFERENCES}

1. Hansson GK, Hermansson A. The immune system in atherosclerosis. Nat Immunol 2011;12:204-212.

2. Finn AV, Nakano M, Narula J, Kolodgie FD, Virmani R. Concept of Vulnerable/Unstable Plaque. Arterioscler Thromb Vasc Biol 2010;30:1282-1292.

3. Ait-Oufella H, Sage AP, Mallat Z, Tedgui A. Adaptive (T and B cells) immunity and control by dendritic cells in atherosclerosis. Circ Res 2014;114:1640-1660.

4. Kawai T, Akira S. The role of pattern-recognition receptors in innate immunity: update on Toll-like receptors. Nat Immunol 2010;11:373-384.

5. Cole JE, Kassiteridi C, Monaco C. Toll-like receptors in atherosclerosis: a 'Pandora's box' of advances and controversies. Trends Pharmacol Sci 2013;34:629-636.

6. Quillard T, Araújo HA, Franck G, Shvartz E, Sukhova G, Libby P. TLR2 and neutrophils potentiate endothelial stress, apoptosis and detachment: implications for superficial erosion. European Heart Journal 2015;36:1394-1404.

7. Salagianni M, Galani IE, Lundberg AM, Davos CH, Varela A, Gavriil A, Lyytikainen LP, Lehtimaki T, Sigala F, Folkersen L, Gorgoulis V, Lenglet S, Montecucco F, Mach F, Hedin U, Hansson GK, Monaco C, Andreakos E. Toll-like receptor 7 protects from atherosclerosis by constraining "inflammatory" macrophage activation. Circulation 2012;126:952-962.

8. Persson J, Folkersen L, Ekstrand J, Helleberg J, Gabrielsen A, Lundman P, Hedin U, Paulsson-Berne G. High plasma adiponectin concentration is associated with all-cause mortality in patients with carotid atherosclerosis. Atherosclerosis 2012;225:491-496.

9. Perisic L, Aldi S, Sun Y, Folkersen L, Razuvaev A, Roy J, Lengquist M, Åkesson S, Wheelock CE, Maegdefessel L, Gabrielsen A, Odeberg J, Hansson GK, Paulsson-Berne 
G, Hedin U. Gene expression signatures, pathways and networks in carotid atherosclerosis. Journal of Internal Medicine 2016;279:293-308.

10. Folkersen L, Persson J, Ekstrand J, Agardh HE, Hansson GK, Gabrielsen A, Hedin U, Paulsson-Berne G. Prediction of ischemic events on the basis of transcriptomic and genomic profiling in patients undergoing carotid endarterectomy. Mol Med 2012;18:669-675.

11. Merlo J, Lindblad U, Pessah-Rasmussen H, Hedblad B, Rastam J, Isacsson SO, Janzon L, Rastam L. Comparison of different procedures to identify probable cases of myocardial infarction and stroke in two Swedish prospective cohort studies using local and national routine registers. Eur J Epidemiol 2000;16:235-243.

12. Tunstall-Pedoe H, Kuulasmaa K, Amouyel P, Arveiler D, Rajakangas AM, Pajak A. Myocardial infarction and coronary deaths in the World Health Organization MONICA Project. Registration procedures, event rates, and case-fatality rates in 38 populations from 21 countries in four continents. Circulation 1994;90:583-612.

13. Irizarry RA, Hobbs B, Collin F, Beazer-Barclay YD, Antonellis KJ, Scherf U, Speed TP. Exploration, normalization, and summaries of high density oligonucleotide array probe level data

10.1093/biostatistics/4.2.249. Biostat 2003;4:249-264.

14. Therneau TM, Grambsch PM. Modeling Survival Data: Extending the Cox Model Springer-Verlag New York, 2000.

15. Perisic L, Hedin E, Razuvaev A, Lengquist M, Osterholm C, Folkersen L, Gillgren P, Paulsson-Berne G, Ponten F, Odeberg J, Hedin U. Profiling of Atherosclerotic Lesions by Gene and Tissue Microarrays Reveals PCSK6 as a Novel Protease in Unstable Carotid Atherosclerosis. Arteriosclerosis, Thrombosis, and Vascular Biology 2013;33:2432-2443. 
16. Koltsida O, Karamnov S, Pyrillou K, Vickery T, Chairakaki AD, Tamvakopoulos C, Sideras P, Serhan CN, Andreakos E. Toll-like receptor 7 stimulates production of specialized pro-resolving lipid mediators and promotes resolution of airway inflammation. EMBO Mol Med 2013;5:762-775.

17. Dominguez-Villar M, Gautron A-S, de Marcken M, Keller MJ, Hafler DA. TLR7 induces anergy in human CD4+ T cells. Nat Immunol 2015;16:118-128.

18. Foks AC, Lichtman AH, Kuiper J. Treating Atherosclerosis With Regulatory T Cells. Arteriosclerosis, Thrombosis, and Vascular Biology 2015;35:280-287.

19. Nilsson J, Lichtman A, Tedgui A. Atheroprotective immunity and cardiovascular disease: therapeutic opportunities and challenges. Journal of Internal Medicine 2015;278:507-519.

20. Mallat Z, Heymes C, Ohan J, Faggin E, Lesèche G, Tedgui A. Expression of Interleukin10 in Advanced Human Atherosclerotic Plaques: Relation to Inducible Nitric Oxide Synthase Expression and Cell Death. Arteriosclerosis, Thrombosis, and Vascular Biology 1999;19:611-616.

21. Han $\mathrm{X}$, Boisvert WA. Interleukin-10 protects against atherosclerosis by modulating multiple atherogenic macrophage function. Thrombosis and Haemostasis 2015;113:505-512.

22. Subramanian M, Thorp E, Tabas I. Identification of a Non-Growth Factor Role for GMCSF in Advanced Atherosclerosis: Promotion of Macrophage Apoptosis and Plaque Necrosis Through IL-23 Signaling. Circulation Research 2015;116:e13-e24.

23. Green NM, Moody K-S, Debatis M, Marshak-Rothstein A. Activation of Autoreactive B Cells by Endogenous TLR7 and TLR3 RNA Ligands. Journal of Biological Chemistry 2012;287:39789-39799. 
24. Clement M, Guedj K, Andreata F, Morvan M, Bey L, Khallou-Laschet J, Gaston A-T, Delbosc S, Alsac J-M, Bruneval P, Deschildre C, Le Borgne M, Castier Y, Kim H-J, Cantor H, Michel J-B, Caligiuri G, Nicoletti A. Control of the T Follicular HelperGerminal Center B-Cell Axis by CD8+ Regulatory T Cells Limits Atherosclerosis and Tertiary Lymphoid Organ Development. Circulation 2015;131:560-570. 
Table 1. Baseline characteristics-

\begin{tabular}{|c|c|c|c|c|c|c|c|c|}
\hline (5) & & Microarra & & & & qPCR & & \\
\hline & TLR7-low & TLR7-medium & TLR7-high & $\mathbf{P}$ & TLR7-low & TLR7-medium & TLR7-high & $\mathbf{P}$ \\
\hline $\mathbf{n}$ & 44 & 44 & 45 & & 41 & 40 & 42 & \\
\hline Male, \% (n) & $78.6(33)$ & $81.8(36)$ & $75.6(34)$ & 0.37 & $82.9(34)$ & $60(24)$ & $87.8(36)$ & 0.78 \\
\hline Age (years) & $73[68.2,76]$ & $74[66.5,78]$ & $71[65,77]$ & 0.36 & $72[66,77]$ & $70[64.5,76.2]$ & $70[64,78]$ & 0.82 \\
\hline Body mass index $\left(\mathrm{kg} / \mathrm{m}^{2}\right)$ & $26[23.7,27.5]$ & $25[23.4,27.9]$ & $25[23.1,26.8]$ & 0.8 & $25[22.6,27.7]$ & $27[24.5,28.6]$ & $26[24.2,28.7]$ & 0.58 \\
\hline Smoking \% (n) & $69.4(25)$ & $57.5(23)$ & $52.4(22)$ & 0.52 & $52.9(18)$ & $54.1(20)$ & $55(22)$ & 0.5 \\
\hline Stenosis \% & $80[73.8,80]$ & $80[75,80]$ & $80[78.8,80]$ & 0.34 & $80[77.5,80]$ & $80[80,80]$ & $80[77.5,80]$ & 0.46 \\
\hline Total cholesterol $(\mathrm{mmol} / \mathrm{L})$ & $4.2[3.8,5.4]$ & $4.6[3.9,5.4]$ & $4.6[4.1,5.3]$ & 0.56 & $4.2[3.6,5.1]$ & $4.3[4,5.4]$ & $4.6[3.77,5.32]$ & 0.5 \\
\hline LDL (mmol/L) & $2.5[2.3,3.65]$ & $2.7[2.08,3.5]$ & $2.4[2.18,3]$ & 0.18 & $2.2[1.9,2.77]$ & $2.3[1.9,3.05]$ & $2.4[1.87,3.23]$ & 0.37 \\
\hline Statins \% (n) & $87.5(35)$ & $70.5(31)$ & $84.4(38)$ & 0.96 & $82.5(33)$ & $82.5(33)$ & $85(34)$ & 0.7 \\
\hline Antihypertensives \% (n) & $81.8(36)$ & $68.2(30)$ & $93.3(42)$ & 0.07 & $78(32)$ & $87.5(35)$ & $90.5(38)$ & 0.17 \\
\hline
\end{tabular}




\begin{tabular}{|c|c|c|c|c|c|c|c|c|}
\hline Antiplatelet agents \% (n) & $85(34)$ & $93.2(41)$ & $93.3(42)$ & 0.63 & $95.1(39)$ & $90(36)$ & $95.1(39)$ & 0.94 \\
\hline Symptomatic \% (n) & $76.2(32)$ & $81.8(36)$ & $73.3(33)$ & 0.97 & $61(25)$ & $77.5(31)$ & $70.7(29)$ & 0.48 \\
\hline Diabetes \% (n) & $22.7(10)$ & $25(11)$ & $26.7(12)$ & 0.56 & $24.4(10)$ & $32.5(13)$ & $19.5(8)$ & 0.35 \\
\hline hsCRP (mg/L) & $2.1[0.94,6.2]$ & $2.5[1.2,6.9]$ & $3.2[1.3,7]$ & 0.59 & $2.9[1.6,5.6]$ & $2.7[1,4.9]$ & $2.2[1.2,7.6]$ & 0.95 \\
\hline
\end{tabular}

Baseline data for microarray and qPCR cohorts categorized on the basis of tertiles of TLR7 expression. Categorical data are reported as percentages (count), and numerical data are expressed as median [Q1, Q3]. P-values were calculated using a linear regression model on the numerical value of the TLR7 expression. The term symptomatic refers to if the patients have had a transient ischemic attack, amaurosis fugax or minor ischemic stroke before the carotid artery surgery. hsCRP stands for high sensitive CRP, LDL for Low-density lipoprotein cholesterol. 
Table 2. Relationship between gene expression levels in carotid artery plaques of TLR7 and outcome.

\begin{tabular}{|c|c|c|c|c|}
\hline & \multicolumn{2}{|c|}{$\begin{array}{l}\text { Major adverse cardio- or } \\
\text { cerebrovascular event }\end{array}$} & \multicolumn{2}{|c|}{ Ischaemic event } \\
\hline & $\begin{array}{l}\text { Microarray } \\
(n=123)\end{array}$ & $\begin{array}{c}\text { qPCR } \\
(n=133)\end{array}$ & $\begin{array}{c}\text { Microarray } \\
(n=123)\end{array}$ & $\begin{array}{c}\text { qPCR } \\
(n=133)\end{array}$ \\
\hline $\begin{array}{l}\text { TLR7, } \\
\text { unadjusted }\end{array}$ & $\begin{array}{l}2.38(\mathrm{P}=0.012) \\
\text { CI 1.21-4.67 }\end{array}$ & $\begin{array}{l}1.91(\mathrm{P}=0.027) \\
\text { CI 1.08-3.4 }\end{array}$ & $\begin{array}{l}2.28(\mathrm{P}=0.044) \\
\text { CI 1.02-5.09 }\end{array}$ & $\begin{array}{l}1.67(\mathrm{P}=0.145) \\
\text { CI } 0.84-3.31\end{array}$ \\
\hline $\begin{array}{l}\text { TLR7, adj. for } \\
\text { age }\end{array}$ & $\begin{array}{l}2.2(\mathrm{P}=0.023) \\
\text { CI 1.12-4.34 }\end{array}$ & $\begin{array}{l}1.91(\mathrm{P}=0.027) \\
\text { CI 1.08-3.39 }\end{array}$ & $\begin{array}{l}2.10(\mathrm{P}=0.072) \\
\text { CI } 0.936-4.69\end{array}$ & $\begin{array}{l}1.63(\mathrm{P}=0.163) \\
\text { CI } 0.82-3.24\end{array}$ \\
\hline $\begin{array}{l}\text { TLR7, adj. for } \\
\text { sex }\end{array}$ & $\begin{array}{l}2.33(\mathrm{P}=0.014) \\
\text { CI 1.18-4.59 }\end{array}$ & $\begin{array}{l}1.87(\mathrm{P}=0.033) \\
\text { CI 1.05-3.32 }\end{array}$ & $\begin{array}{l}2.24(\mathrm{P}=0.049) \\
\text { CI } 1-5.02\end{array}$ & $\begin{array}{l}1.63(\mathrm{P}=0.162) \\
\text { CI } 0.82-3.25\end{array}$ \\
\hline $\begin{array}{l}\text { TLR7, adj. for } \\
\text { LDL }\end{array}$ & $\begin{array}{l}2.35(\mathrm{P}=0.014) \\
\text { CI 1.19-4.64 }\end{array}$ & $\begin{array}{l}1.90(\mathrm{P}=0.028) \\
\text { CI } 1.07-3.38\end{array}$ & $\begin{array}{l}2.20(\mathrm{P}=0.057) \\
\text { CI } 0.978-4.93\end{array}$ & $\begin{array}{l}1.65(\mathrm{P}=0.155) \\
\text { CI } 0.83-3.28\end{array}$ \\
\hline $\begin{array}{l}\text { TLR7, adj. for } \\
\text { age and sex }\end{array}$ & $\begin{array}{l}2.18(\mathrm{P}=0.024) \\
\text { CI 1.11-4.30 }\end{array}$ & $\begin{array}{l}1.82(\mathrm{P}=0.042) \\
\text { CI 1.02-3.23 }\end{array}$ & $\begin{array}{l}2.07(\mathrm{P}=0.076) \\
\text { CI 0.93-4.63 }\end{array}$ & $\begin{array}{l}1.57(\mathrm{P}=0.199) \\
\text { CI } 0.79-3.13\end{array}$ \\
\hline $\begin{array}{l}\text { TLR7, adj. for } \\
\text { age, sex and } \\
\text { LDL }\end{array}$ & $\begin{array}{l}2.2(\mathrm{P}=0.024) \\
\text { CI } 1.11-4.35\end{array}$ & $\begin{array}{l}1.80(\mathrm{P}=0.045) \\
\text { CI 1.01-3.21 }\end{array}$ & $\begin{array}{l}2.02(\mathrm{P}=0.090) \\
\text { CI } 0.90-4.56\end{array}$ & $\begin{array}{l}1.55(\mathrm{P}=0.214) \\
\text { CI } 0.78-3.09\end{array}$ \\
\hline
\end{tabular}

Hazard ratio (HR) (95\% confidence interval, P-values) from Cox regression models for the lowest tertile of TLR7 expression versus the middle and highest combined. 


\section{LEGENDS}

Figure 1.Worse outcome were found for patients with lower levels of TLR 7 transcript in their plaques. Kaplan-Meier curve illustrating MACCE-free survival of patients with TLR7 expression of highest tertile (black), the middle tertile (dark grey) and the lowest tertile (light grey). A) Microarray cohort with 34 MACCE and 24 ischaemic event. B) qPCR cohort with 47 MACCE and 33 ischaemic events. Below each plot, the count of event-free patients is indicated. The $\mathrm{x}$-axis scale of the study was time since CEA in months. Hazard ratio (HR) for the lowest tertile of TLR7 expression versus the middle and highest tertiles combined from a Cox regression is reported in the upper-right corner.

Figure 2. Immunohistochemical staining of carotid plaques for TLR7 and cell markers. (a, b) TLR7 staining in a T cell-rich area. (c) CD3-positive staining in consecutive slides. (d) CD8 staining in consecutive slides. (e, f) TLR7. (g) CD68. (h) CD163. Positive cells are stained with Vulcan fast red, and the nuclei are stained blue. Scale bars: (a, f) $100 \mu \mathrm{m}$. (b-d, f-g) 10 $\mu \mathrm{m}$.

Figure 3. Co-localization of TLR7 with macrophage and endothelial cell markers in human carotid lesions detected by immunofluorescence staining. (a, b) TLR7 (red) colocalization with the macrophage marker CD68 (green). (c, d) Co-expression of TLR7 with the macrophage marker CD163 (green). Differential expression of TLR7 in endothelial cells. (e, f) No co-expression of TLR7 with lumen endothelial cells (vWF, green). (g, h) Co-localization of TLR7 with capillary endothelial cells (vWF, green). Nuclei are stained blue. Scale bars: (a-f) $7.5 \mu \mathrm{m},(\mathrm{g}-\mathrm{h}) 25 \mu \mathrm{m}$.

Figure 4. Co-localization of TLR7 and $T$ cell markers in lesions detected by immunofluorescence staining. (a-d) TLR7 (red) is co-expressed in CD4+ and CD8+ (green) cells. CD4 T cells are labelled with arrows (CD3+ CD8-). (e-h) CD3 (grey), CD69 (green) and 
triple staining of CD3+, TLR7 and the T cell activation marker CD69. (i-1) CD3+ cells, FoxP3 cells (green) and triple staining of TLR7, CD3, and FoxP3. Nuclei are stained blue. Scale bars: $7.5 \mu \mathrm{m}$.

Figure 5. Ex vivo dose-dependent cytokine secretion in human carotid plaques after TLR7 stimulation. Fresh plaques were used at the same day of operation. Minced plaque tissue was incubated in vitro with increasing concentration of TLR7 ligand imiquimod. IL-10, GM-CSF, TNF- $\alpha$ and IL-12/IL-3p40 secretion significantly increased in a dose-dependent manner after $20 \mathrm{~h}$ of stimulation with $2.5,5$, and $12.5 \mu \mathrm{g}$ IMQ, measured by multiplex ELISA of total 20 cytokines. For each cytokine, fixed-effects linear regression was used to test for a linear trend between IMQ stimulation and mean cytokine secretion. P-values were adjusted for multiple comparisons of 20 cytokines using Bonferroni correction. Number of plaques analysed $n=12$. Summary of all cytokine data in table S2.

Figure 6. Reduction of cytokine secretion in human carotid plaques after inhibition of TLR7. Fresh plaques were used at the same day of operation. Minced plaque tissue was incubated in vitro with optimal concentration of TLR7 ligand in presence or absence of TLR7 inhibitor. IL-10, GM-CSF, TNF- $\alpha$ secretion was strongly decreased after the addition of $5 \mu \mathrm{M}$ TLR7 inhibitor (ODN 20958) and $12.5 \mu \mathrm{g} / \mathrm{ml}$ IMQ. Number of plaques analysed $\mathrm{n}=8$. Data presented as mean \pm SD. For each cytokine one-way paired ANOVA followed by Bonferroni post hoc tests was used to compare the different groups.

Figure 7. Co-localization of IL-10 with macrophage, $T$ cell and smooth muscle cell markers after ex vivo stimulation with IMQ. Localization of IL-10 secreting cells after $e x$ vivo short term stimulation of plaque tissue with TLR7 ligand. a) IL-10 (red) and the M2 macrophage marker CD163 (green). b) IL-10 (red) co-expression with CD3 (green). c) IL-10 (red) co-localization with a-actin (green). Nuclei are stained blue. Scale bars: $10 \mu \mathrm{m}$. 
Figure 1

A) Microarray

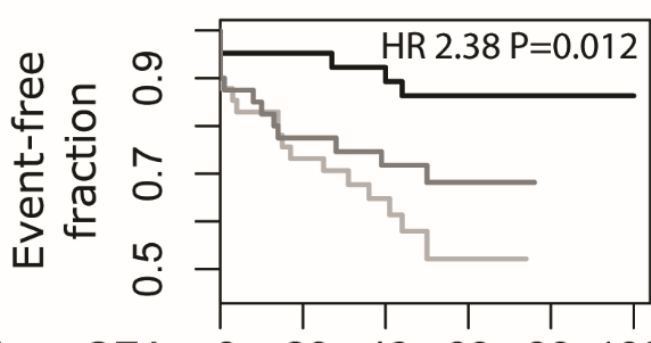

$\begin{array}{lllllll}\text { Months from CEA } & 0 & 20 & 40 & 60 & 80 & 100\end{array}$

High-TLR7

Medium-TLR7

Low-TLR7 $\begin{array}{llllll}42 & 40 & 31 & 17 & 6 & 1\end{array}$

$\begin{array}{llllll}40 & 31 & 25 & 9 & 0 & 0\end{array}$

$\begin{array}{llllll}41 & 30 & 19 & 4 & 0 & 0\end{array}$
B) $\quad \mathrm{qPCR}$

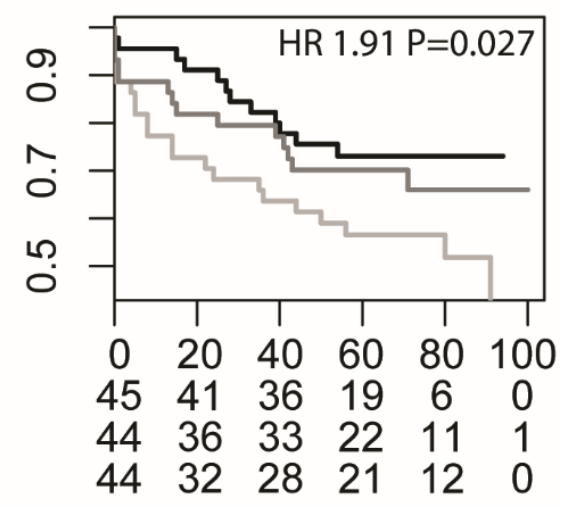


Figure 2

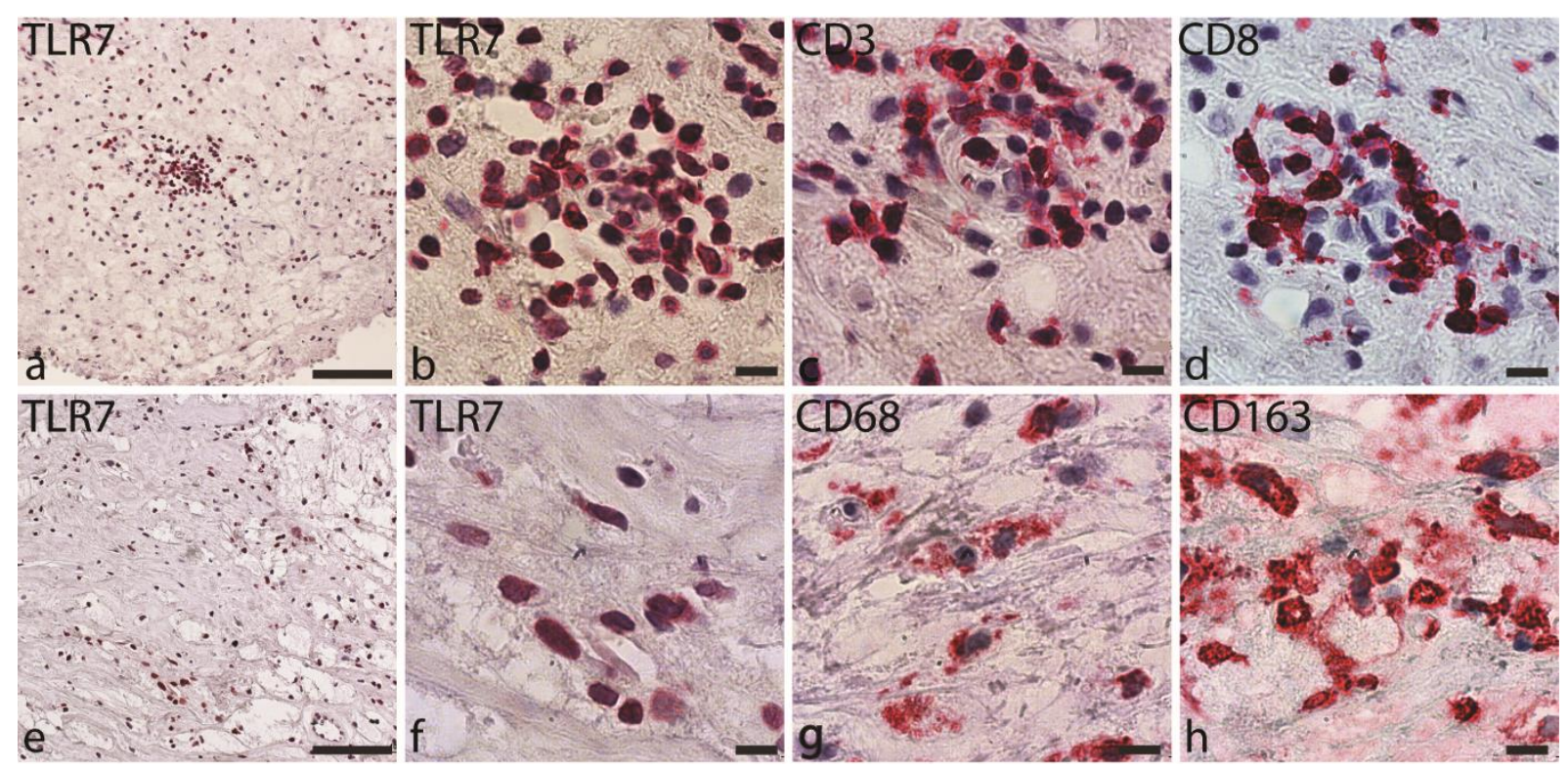


Figure 3

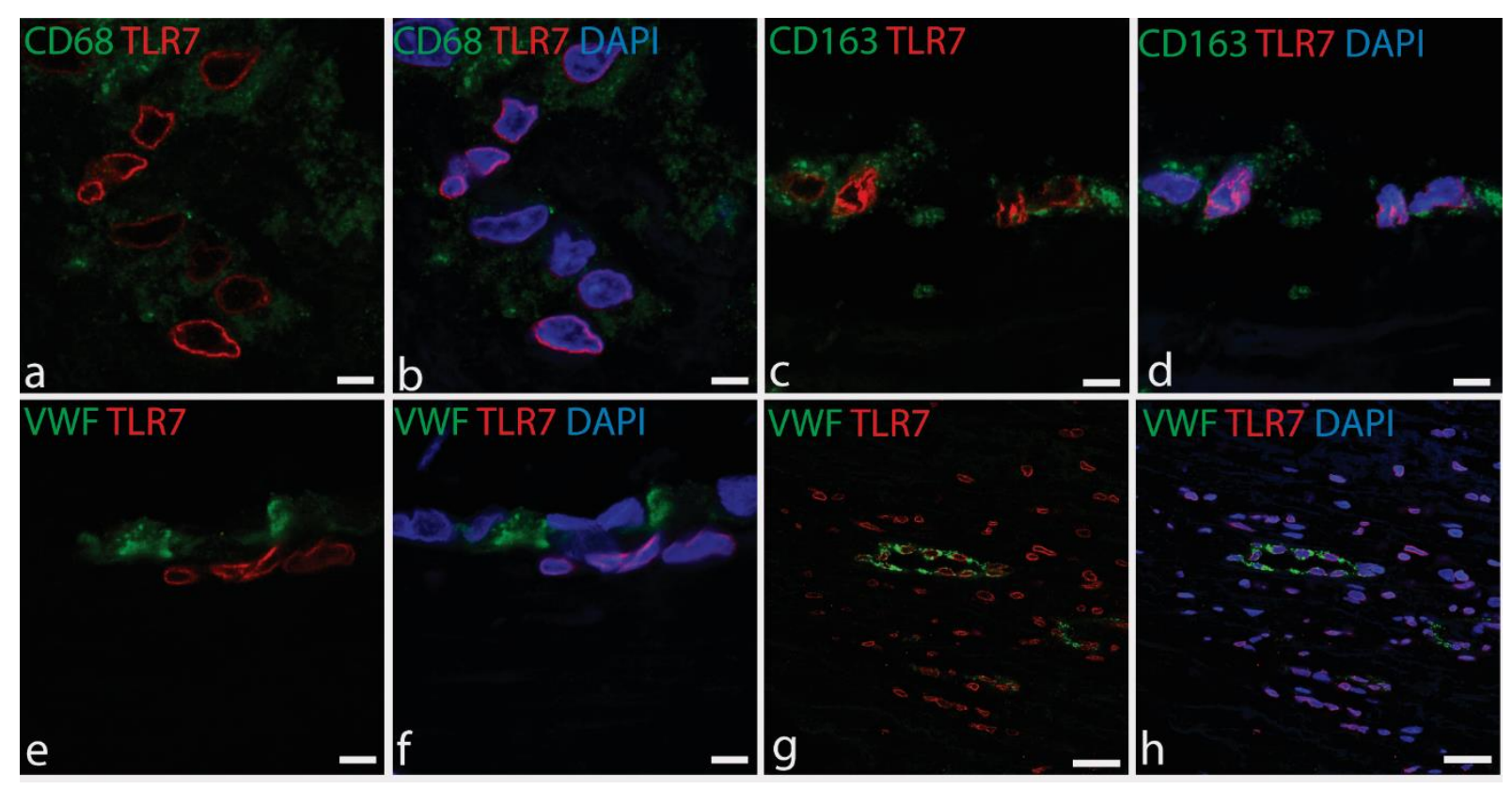


Figure 4

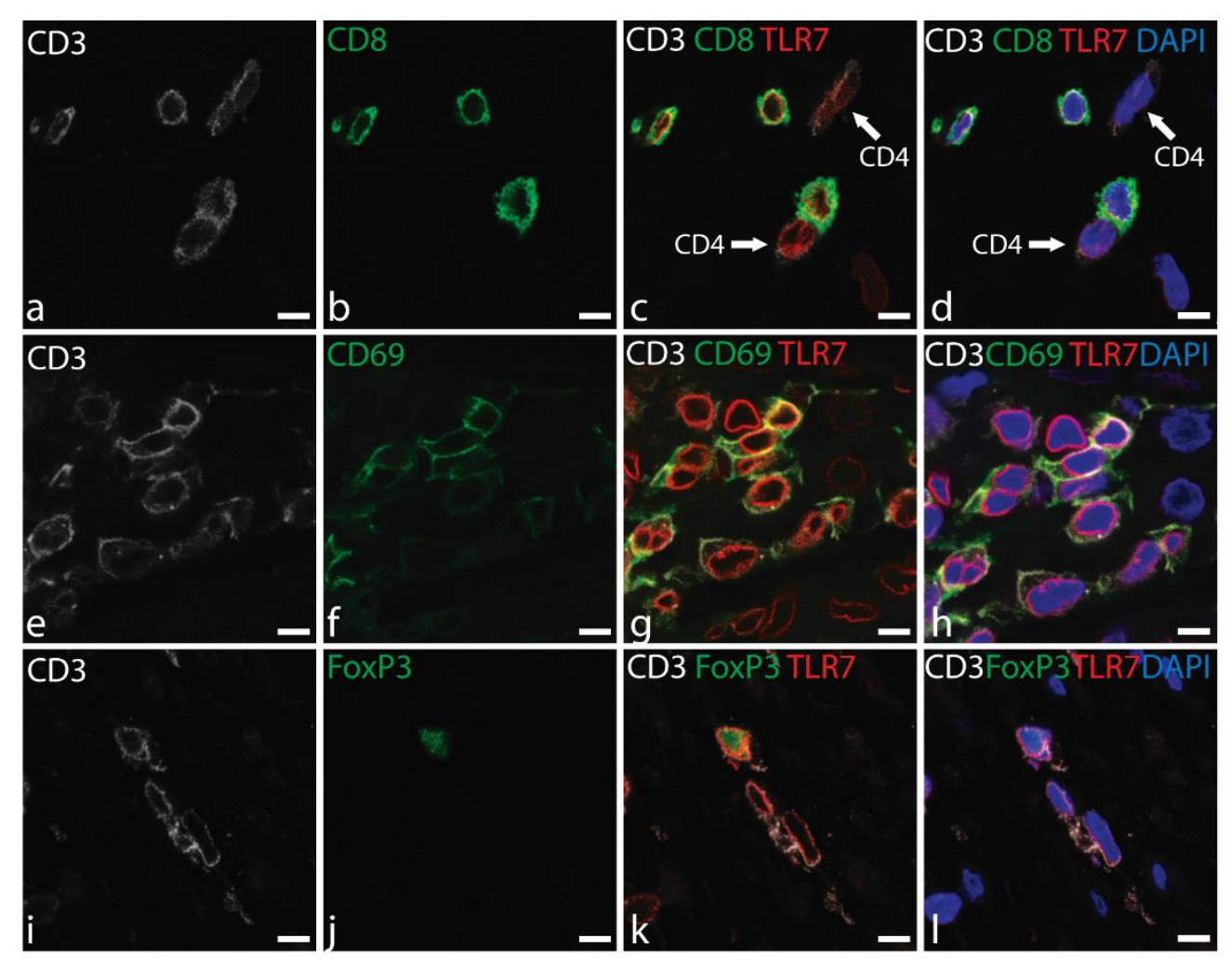


Figure 5
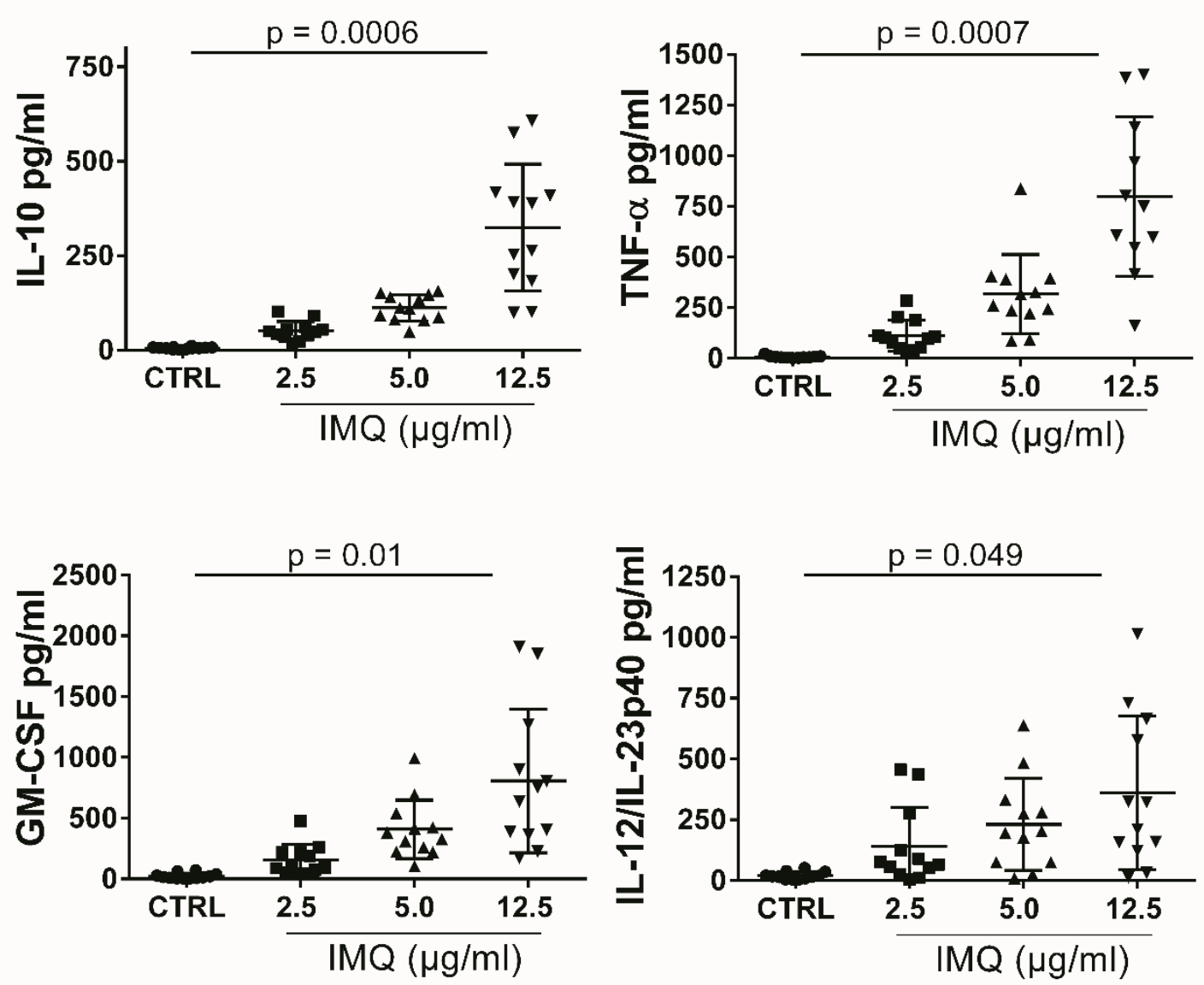
Figure 6
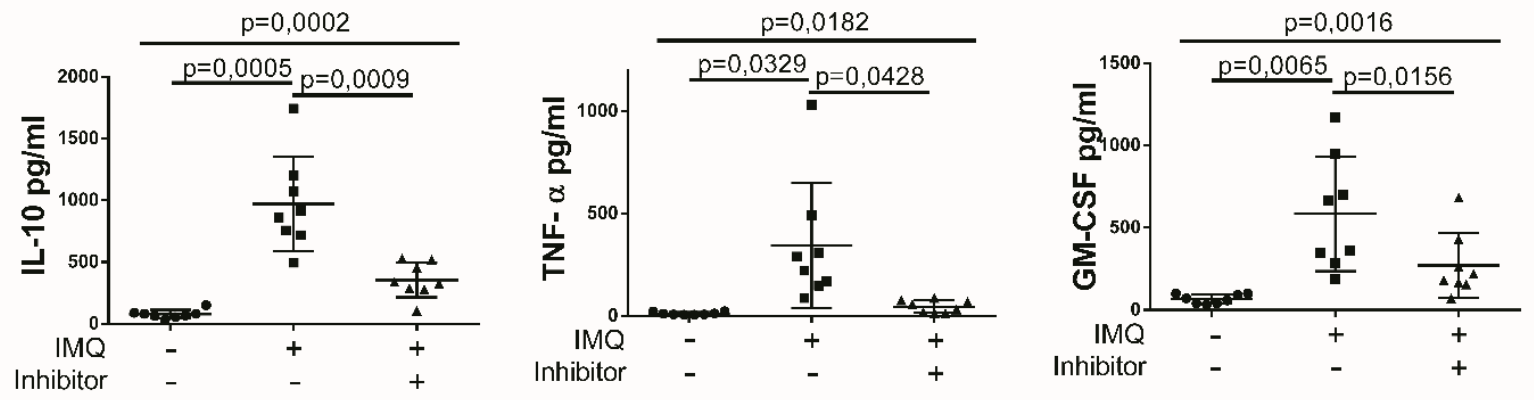
Figure 7

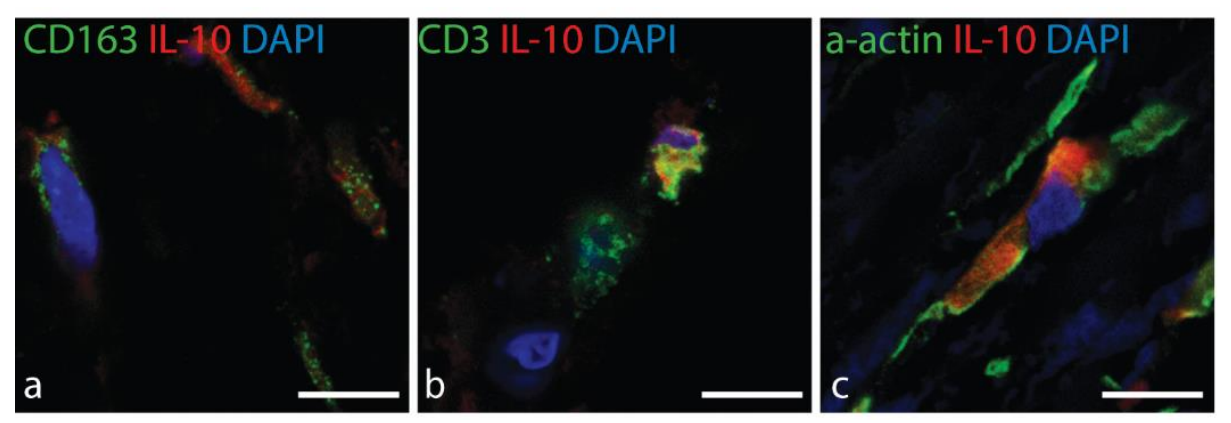

